\title{
Coupled Deconvolution for Frequency Extrapolation of Electromagnetic Solutions with Matrix Pencil Method ${ }^{\dagger}$
}

\author{
Levent Gürel* and Ferhat Yıldırım \\ Department of Electrical and Electronics Engineering \\ Bilkent University, TR-06800, Bilkent, Ankara, Turkey \\ E-mail: lgurel@ee.bilkent.edu.tr, ferhat@ee.bilkent.edu.tr
}

\begin{abstract}
Matrix pencil method (MPM) has been widely used to estimate the parameters of complex-exponential based models. An important application is the extrapolation of the frequency-domain solutions of electromagnetic problems. In this paper, we present a mathematical tool, namely, coupled deconvolution, which improves the performance of the MPMbased extrapolation of electromagnetic solutions.
\end{abstract}

\section{INTRODUCTION}

Solutions of large geometries at high frequencies have always been of interest in computational electromagnetics. Accurate solutions of these problems demand more memory and CPU time as the solution frequency increases. For very high frequencies, computationally inexpensive high-frequency prediction techniques can be used to obtain satisfactory solutions. Usually, accurate solution intervals of these two classes of computational solvers do not coincide. In this work, we propose using frequency extrapolation to estimate the solution for the intermediate frequency band.

Various numerical approaches have been developed to increase the solution efficiency of the frequency-domain electromagnetic solvers. Fast methods, such as fast multipole method (FMM) and multi-level fast multipole algorithm (MLFMA), and complex solution strategies, such as parallel computing, have helped tremendously to push the frequency limits higher; nevertheless, the limits exist. High-frequency solution techniques, such as physical optics (PO), based on the high-frequency approximations of electromagnetics, give accurate solutions for relatively high-frequencies but the accuracy decreases as the solution frequency decreases.

In this work, we use model-based extrapolation approach to estimate the solution data in the intermediate frequency band. This is performed by first constructing a signal model from the known part of the signal. This model, which is an approximation to the known signal, is assumed to be valid also for the unknown part of the signal. Extrapolation is performed by evaluating the constructed model for the frequencies in the unknown part of the signal. To construct this model, due to its noise tolerance and computational efficiency, we use the matrix pencil method (MPM) [1].

When performing the extrapolation, we have two alternatives to find the estimated scattering solution for the intermediate frequencies. In the forward case, we model the available lowfrequency data and perform the extrapolation in the increasing frequency direction. In the

\footnotetext{
${ }^{\dagger}$ This work was supported by the Turkish Academy of Sciences in the framework of the Young Scientist Award Program (LG/TUBA-GEBIP/2002-1-12), by the Scientific and Technical Research Council of Turkey (TUBITAK) under Research Grant 103E008, and by contracts from ASELSAN and SSM.
} 
backward case, the available high-frequency data is modelled and extrapolation is performed in the decreasing frequency direction.

\section{COUPLED DECONVOLUTION IN MPM EXTRAPOLATION}

In the forward and backward MPM extrapolation, as will be presented in the next section, the error levels compared to the reference data are low in the beginning of the extrapolation region. However, the error tends to increase as we go further in frequency in the extrapolation direction. This behavior is observed for both forward and backward cases. In this work, we propose using coupled deconvolution to combine these two separate extrapolation cases, which will give us a single extrapolation signal with lower error levels. Deconvolution is simply defined as undoing what convolution does. It is a well known property that the convolution operation in the time domain corresponds to multiplication in the Fourier domain [2]. In discrete domain, this relation can be represented as

$$
c[n]=\mathrm{DFT}^{-1}\{\mathrm{DFT}\{x[n]\} \cdot \operatorname{DFT}\{y[n]\}\},
$$

where DFT stands for $N$-point discrete fourier transform. $c[n]$ in (1) can also be calculated in time domain by

$$
c[n]=\sum_{m=0}^{N-1} x\left[\langle m\rangle_{N}\right] y\left[\langle n-m\rangle_{N}\right] .
$$

Equation (2) defines an $N$-point circular convolution, where $\langle.\rangle_{N}$ defines a circular shift in a length $N$ sequence. With this set of signals, finding $x$ from known $c$ and $y$ is a circular deconvolution problem. If we rewrite (2) in matrix notation,

$$
[Y][\bar{x}]=[\bar{c}],
$$

we can define the circular deconvolution problem as

$$
[\bar{x}]=[Y]^{-1}[\bar{c}],
$$

where $[Y]$ is $N \times N$ circular convolution matrix that contains the values of $y$.

Suppose the complete solution signal of an electromagnetic problem is $Y$. From low-frequency solvers we obtain the $\left(0, f_{L}\right)$ interval and from high-frequency solvers we obtain the $\left(f_{H}, f_{\max }\right)$ interval. By using forward MPM extrapolation, we can estimate $\left(f_{L}, f_{\max }\right)$ interval and by using backward MPM extrapolation, we can estimate $\left(0, f_{H}\right)$ interval. We define the coupling region as $\left(f_{L}, f_{H}\right)$ and combine the two extrapolation results to achieve a lower error level.

We first define two window functions in the solution domain: $W_{L}$, which has nonzero values in the interval $\left(0, f_{H}\right)$, and $W_{H}$, which has nonzero values in the interval $\left(f_{L}, f_{\max }\right)$. Fig. 1 shows two Chebyshev windows that are constructed in this manner. Forward MPM extrapolation is performed up to $f_{H}$ to obtain $X_{L}$; backward MPM extrapolation solution is performed down to $f_{L}$ to obtain $X_{H}$. By using these four known signals, we can write two equations that contain the unknown signal $Y$ as

$$
\begin{gathered}
W_{L} \cdot Y=X_{L}, \\
W_{H} \cdot Y=X_{H} .
\end{gathered}
$$

In time domain, we can rewrite (5) and (6) in matrix form as

$$
\left[W_{L}\right][\bar{y}]=\left[\bar{x}_{L}\right],
$$




$$
\left[W_{H}\right][\bar{y}]=\left[\bar{x}_{H}\right],
$$

where $\left[W_{L}\right]$ and $\left[W_{H}\right]$ are the circular convolution matrices of $\left[w_{L}\right]$ and $\left[w_{H}\right]$, respectively. If we combine (7) and (8) into single matrix equation, then

$$
\left[\begin{array}{l}
{\left[W_{L}\right]} \\
{\left[W_{H}\right]}
\end{array}\right][\bar{y}]=\left[\begin{array}{l}
\bar{x}_{L} \\
\bar{x}_{H}
\end{array}\right] .
$$

Equation (9) is a total least-squares problem, whose solution will approximate the time-domain counterpart of the solution signal $Y$ of the electromagnetic problem. Various approaches can be used to solve (9), such as singular value decomposition or pseudoinverse [3].

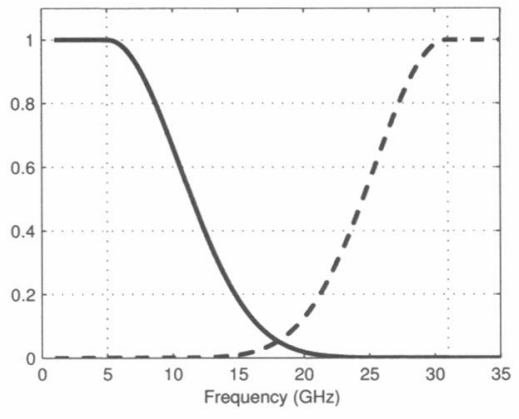

Fig. 1. Chebyshev windows used in coupled deconvolution.

\section{NUMERICAL EXAMPLE}

In order to demonstrate the improvement induced by the coupled deconvolution on the MPM extrapolation, we will present the problem of scattering from a conducting sphere. Backscattering of the conducting sphere under plane-wave illumination has analytical solution, hence we can quantitatively determine the improvement on the accuracy of the extrapolation supplied by the coupled deconvolution. .

Using the analytical Mie-series solution, we obtain the backscattering solution of a conducting sphere with a $30 \mathrm{~cm}$ radius between $1-35 \mathrm{GHz}$. First, $1-5 \mathrm{GHz}$ band of this data is assumed to be available as if it is obtained from a numerical solver. At $5 \mathrm{GHz}$, numerical solution of this problem requires a solution of 100,000 unknowns, which makes this bandwidth choice reasonable. MPM model is constructed using this $4 \mathrm{GHz}$ band of data and forward MPM extrapolation is performed up to $35 \mathrm{GHz}$. Fig 2(a) shows the performance of the forward extrapolation in terms of the error between the extrapolated signal and the analytical solution.

Next, 31-35 GHz band of the backscattering data is assumed to be available through a highfrequency approximation technique. This band of data is used to construct the MPM model and backward MPM extrapolation is performed down to $1 \mathrm{GHz}$. Fig 2(b) shows the error of the backward extrapolation.

Finally, by using the windows shown in Fig. 1, these two extrapolation results are coupled. Fig. 3 shows the error of the extrapolation after coupled deconvolution is applied to forward and backward MPM extrapolation results. Considering Figs. 2(a) and 2(b) together, it can be seen that the extrapolation errors for both cases grow as the extrapolation marches away from 
the available data. When these results are compared with Fig. 3, application of the coupled deconvolution clearly improves the error performance.

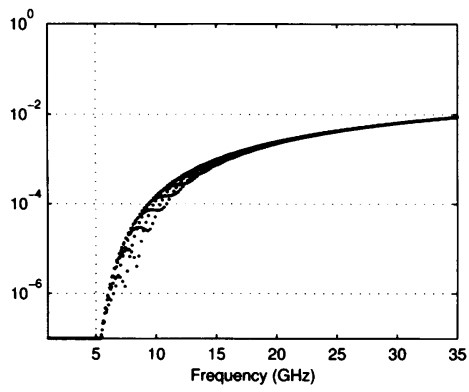

(a)

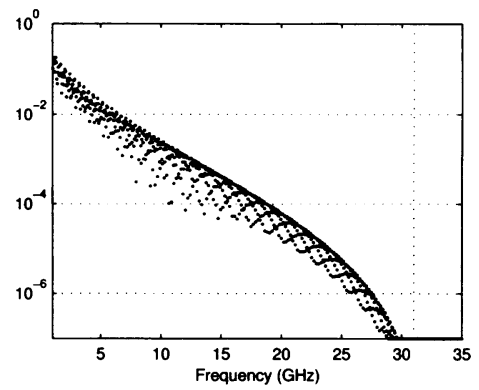

(b)

Fig. 2. Error of (a) forward MPM extrapolation; (b) backward MPM extrapolation.

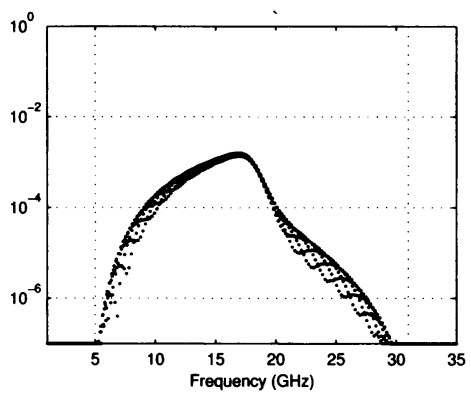

Fig. 3. Error is reduced when coupled deconvolution is used to combine the forward and backward extrapolation schemes.

\section{CONCLUSION}

In this paper, we present a mathematical tool, namely, coupled deconvolution, to be used in conjunction with the MPM extrapolation method for the frequency extrapolation of electromagnetic solutions. Using the low-frequency and high-frequency solutions of an electromagnetic problem, we can successfully estimate the solution in the intermediate frequency band.

\section{REFERENCES}

[1] F. Yıldınm and L. Gürel, "Coupled matrix pencil method for frequency extrapolation of electromagnetic solutions," 2005 IEEE AP-S International Symposium, Washington, DC, July 2005.

[2] Alan V. Oppenheim, Ronald W. Schafer and John R. Buck, Discrete-Time Signal Processing. Upper Saddle River, NJ: Prentice Hall, Inc., 1999.

[3] Gene H. Golub and Charles F. Van Loan, Matrix Computations. Baltimore, MD: Johns Hopkins University Press, 1989. 